\title{
IUna herramienta didáctica para la aplicabilidad del componente pragmático en E/LE
}

\author{
EVA GONZÁLEZ MELÓN
}

KU Leuven

eva.gonzalezmelon@kuleuven.be

\begin{abstract}
Resumen: Enseñar español implica algo más que cubrir contenidos léxico-gramaticales e introducir a los alumnos en su herencia cultural o histórica. Una lengua se inscribe en unas coordenadas pragmático-sociales concretas, algo que tiende a convertirse con frecuencia en una asignatura pendiente durante su enseñanza, dada la subjetivad que entraña su tratamiento. Fomentar que nuestros estudiantes adquieran una competencia pragmático-intercultural —además de una meramente lingüística - empieza a pesar sobre los hombros del profesor de E/LE por falta de herramientas didácticas específicas. Este artículo describe y presenta el funcionamiento del método RICAI que pretende servir de apoyo para la comunidad docente y ofrecer un recurso para la enseñanza del componente pragmático.
\end{abstract}

Palabras clave: Pragmática, interculturalidad, herramienta didáctica.

\section{A didactic tool for the applicability of the pragmatic component in SFL}

Abstract: Teaching Spanish as a foreign language implies more than covering grammatical o lexical components or even introducing the cultural and historic heritage of that country. Forgetting that a language is inscribed in certain socio-pragmatic coordinates often tends to become a pending subject in the teaching of a foreign language, given its subjectivity when it comes to bringing it into the classroom. The need to raise awareness that our students acquire an intercultural competence, in addition to a purely linguistic one, weighs on the shoulders of the Spanish teachers, who claim the elaboration of specific adequate didactic tools. Therefore, the curent theoretical article introduces ans illustrates the functioning of the RICAI model, which aims to provide support to the teaching community and to become a resource for teaching components of pragmatic nature.

Key words: Pragmatics, interculturality, didactic tool.

\section{Introducción}

La existencia de una serie de convenciones socioculturales que no se pueden evidenciar ni omitir impone a los profesores de una lengua extranjera la necesidad de integrarlos en el aula para proveer al alumnado de las herramientas necesarias y que, de este modo, puedan funcionar acorde a ellas. Nuestro compromiso, por tanto, debe ser formar a nuestros alumnos en la interculturalidad (MCERL, 2002: $47^{1}$ e Instituto Cervantes,

\footnotetext{
${ }^{1}$ Según el MCERL (2002) «el alumno [...] se convierte en plurilingüe y desarrolla una interculturalidad. Las competencias lingüística y cultural respecto a cada lengua se modifican mediante el conocimiento de 
2012: 11). Así lo afirma Mendoza Fillola (2003: 11 citado en Trujillo, 2005: 32) cuando se refiere a los objetivos didácticos principales de la enseñanza de lenguas y afirma que «la competencia intercultural implica, según estas definiciones, conocimientos, destrezas y actitudes, es decir, todas las variables que conducen a "formar hablantes competentes y lectores eficaces, buenos redactores, individuos plenamente desarrollados y capaces de relacionarse con los demás" [...]».

Sin embargo, lo que ocurre cuando echamos un vistazo a lo que en realidad sucede en las aulas dista en cierto modo de estas expectativas teóricas, puesto que esos criterios interculturales integran asimismo aspectos de naturaleza (socio)pragmática cuya enseñanza suele ser mínima y, en cierto modo, simplista. Reducir el componente pragmático de nuestra lengua a aspectos como dar dos besos o la mano cuando nos presentan a alguien, llevar un obsequio si nos invitan a comer o utilizar la forma formal 'usted' para mostrar cortesía, no hace sino peligrar la adquisición de esta competencia intercultural. La clave reside en que obviarla privaría a nuestros estudiantes de una competencia necesaria para comunicarse y hacer que ese intercambio comunicativo sea efectivo en un aquí y ahora, con unos interlocutores concretos y una serie de factores extralingüísticos dados por un contexto concreto. Como bien afirma Trujillo Sáez (2005: 33):

La interculturalidad (...) se puede describir en términos estáticos y dinámicos: se describe estáticamente cuando se utiliza para describir una situación comunicativa en la que se ponen en contacto dos (o más) individuos que se perciben el uno al otro como pertenecientes a distintas culturas; se describe dinámicamente cuando se utiliza para describir los mecanismos que se ponen en funcionamiento en esa interacción comunicativa $\mathrm{y}$, especialmente, para que esa comunicación sea efectiva.

En el presente artículo pretendemos, por un lado, (1) revisar brevemente los numerosos intentos llevados a cabo para describir el componente pragmático $(\mathrm{CP}$, a partir de ahora) dentro del aula, así como (2) reflexionar sobre la importancia y la problemática que plantea en E/LE; por otro, (3) presentamos una herramienta que fomenta la aplicabilidad del CP a través de una secuenciación didáctica para su enseñanza en el aula.

\section{El intento semi-fallido de las contribuciones bibliográficas}

Los estudios centrados en el fenómeno de la pragmática como competencia fundamental en la enseñanza de una lengua extranjera, así como del español, son múltiples (Hymes, 1971; Bardovi-Harlig et al., 1991; Bardovi-Harlig y Mahan-Taylor, 2003; Pons Bordería, 2005; Siebold, 2008; Félix-Brasdefer, 2007, 2008; Betancourt Romero, 2012; Shively, 2011; Félix-Brasdefer y Cohen, 2012; Betancourt Romero, 2012; FélixBrasdefer y Koike, 2014; Pinto y De Pablos-Ortega, 2014; Vera Luján y Blanco Rodríguez, 2014; Taguchi, 2009 2011; Van Compernolle, 2014a, 2014b; Van Compernolle et al., 2016; Félix-Brasdefer, 2018, entre otros). Dichos trabajos han contribuido a esclarecer un poco más el tratamiento de este componente que para muchos docentes seguía siendo un perfecto desconocido. La cuestión es que la gran mayoría de ellos se ha limitado a tratarlo desde el punto de vista teórico, dejando a un lado el enfoque más necesario para el profesor dentro del aula que es la cuestión

la otra lengua y contribuyen a crear una conciencia, unas destrezas y unas capacidades interculturales» (MCERL, 2002: 47). 
práctica o aplicada. Es más, la tradición ha centrado sus esfuerzos en el estudio del CP desde una perspectiva fundamentalmente teórico-lingüística -actos del habla, deícticos, marcadores del discurso, etc.- debido al vínculo que mantiene con lo que se supone codificado y a inferir algo que viene condicionado por el contexto de uso; en definitiva, características esencialmente intangibles y difíciles de materializar o categorizar (Gironzetti y Koike, 2017). Si bien estos trabajos han supuesto un reconocido impulso sobre la importancia del estudio del CP, la aplicación de este último en el aula de lenguas extranjeras sigue suponiendo una incógnita. En suma,

we must consider that pragmatics is much more than speech acts and deictic categories. Implicatures and presupposition lie at the heart of pragmatics; they are tied to background knowledge and experience and are related not to forms as much as to context. That is, in order to be taught and understood or produced by L2 students, they require knowledge and experience with the target language and cultural norms, and attention to how all these factors interact with individual personalities (Gironzetti y Koike, 2017: 93).

A pesar de que el CP aparece reconocido como una de las competencias fundamentales en la enseñanza de segundas lenguas y extranjeras (PCIC, 2006; MCERL, Consejo de Europa 2002, 2018) -con el fin de fomentar ese conocimiento intercultural y un entendimiento de la cultura de la nueva lengua meta- continúa faltando una guía práctica de supervivencia para el docente que le permita enseñarlo en el aula.

Más allá de conocer lo que se entiende por pragmática y su fundamental papel a la hora de estudiar una segunda lengua para promover esa competencia intercultural, nos tenemos que plantear por qué supone un pilar fundamental en la enseñanza de una lengua extranjera y, más aún, cómo conseguimos como docentes llevarlo al aula en forma de prácticas docentes que rompan esta barrera entre presupuestos y acción. Gracias a trabajos que van un paso más allá (Álvarez et al., 2006; Toledo, 2012; Leyou, 2015; Gironzetti y Koike, 2017; Brasdefer, 2018; Amenós et al., 2019, entre muchos otros), se empieza a reclamar la necesidad de que existan métodos orientados a su aplicabilidad que nos permitan integrar el CP de una manera realista y gestionarlo de manera adecuada en el aula.

\section{3. un fenómeno considerado de segunda}

La problemática surge cuando nos planteamos la enseñanza de aspectos de origen sociopragmático ${ }^{2}$ como, por ejemplo, los siguientes:

1. Que en España nos pegamos por pagar en un restaurante o en un bar, aun no teniendo dinero.

2. Que perdonamos devolver 50 céntimos o un euro a la hora de hacer cuentas.

3. Que cuando decimos que nos vamos de una cena a la que nos han invitado, ha pasado ya más de una hora y no terminamos de irnos.

4. Que atiborramos a nuestros comensales con cantidades desorbitadas.

5. Que hablamos a gritos transmitiendo un equivocado enfado o una disfrazada mala educación.

\footnotetext{
${ }^{2}$ Aquellos relativos a las expectativas de comportamientos y percepciones sociales de la lengua meta.
} 
6. Que nos cuesta respetar la transición de turnos conversacionales y nos pegamos por hablar.

Todos y cada uno de ellos son un reflejo de nuestra realidad como hispanohablantes. Sin embargo, ¿los enseñamos en clase realmente? ¿Facilitamos como docentes a nuestros estudiantes este tipo de conocimientos además de los meramente lingüísticos? ¿Disponemos del tiempo dentro del plan de estudios y, más importante aún, de las herramientas para llevarlos al aula? En suma, ¿nos hemos planteado su importancia para hacer a nuestros estudiantes partícipes de la interculturalidad? Sin ir más lejos, consideremos (1):

(1) A: ¿Qué tal?

B: Pues nada, ya ves, aquí tirando

La cantidad de emisiones de enunciados como la intervención reactiva del interlocutor $\mathrm{B}$ en (1) son en nuestra lengua extremadamente frecuentes y quedan relegados a un cajón de sastre. Admitamos que intentar enseñar este tipo de intervenciones propias del discurso oral es de gran complejidad dado su vago contenido semántico, pero altamente pragmático. Ahora bien, ¿qué hacemos con este tipo de enunciados? No cabe duda de que «forms are easy to pick up, but sociopragmatic meanings are difficult to deduce without someone making the patterns visible to learners» (Van Compernolle et al., 2016: 343). De hecho, la carga pragmática que subyace a cualquier enunciado emitido por un interlocutor a otro constituye un foco instruccional problemático a la hora de llevar ciertos componentes de la lengua al aula. Con frecuencia, nos quedamos en un nivel superficial limitando inconscientemente a nuestros estudiantes la adquisición del CP. Tomemos, por ejemplo, el caso de las construcciones imperativas en español. Simplificar la enseñanza de su uso para expresar mandatos, órdenes, invitaciones $\mathrm{u}$ ofrecimientos ${ }^{3}$ y con una tendencia a ser percibidos como poco corteses, dista mucho de la realidad intencional con la que los nativos las utilizamos. Sus funciones pragmáticas son más extensas, a saber: petición como en (2) y (3) o sugerencia (4), frecuentemente en compañía de un elemento mitigador -'anda' en (2), 'cuando puedas' en (3) o una justificación de carácter explicativo del tipo 'no vaya a ser que cojas frío' como en (4)-. Todas y cada una de ellas vendrán dadas por el contexto y una serie de factores extralingüísticos a tener en cuenta, lo que pone en tela de juicio esa carga descortés que se suele otorgar al uso de esta construcción gramatical.

(2) Préstame 10 euros, anda

(3) Ponme un café, cuando puedas

(4) Ponte el abrigo, no vaya a ser que cojas frío

La enseñanza de estos aspectos de la lengua supone, sin duda, una complejidad que pone a prueba al docente de una lengua extranjera, pero que no por ello han de ser ignorados. Dejarlos de lado no hace más que potenciar la producción de posibles

\footnotetext{
3 Según Fernández Silva (2002: 6) «es falso que "en España el imperativo se usa mucho y que es suficiente agregar un por favor por razones de cortesía”. En España, el uso del imperativo es habitual, pero no en peticiones sino en invitaciones y ofrecimientos (vente al cine con nosotros, siéntate, mujer, y ponte cómoda, sírvete un poco más), en concesión de permiso (pase, pase; coja lo que quiera) y en usos cristalizados que pueden adquirir diferentes significados como llamar la atención del interlocutor (oiga, fíjate), o de expresión de incredulidad (venga ya)" ». No estamos de acuerdo con el autor en lo que al uso del imperativo para expresar peticiones informales se refiere.
} 
interferencias pragmáticas provocadas por transferencia o interferencia de comportamientos sociales de la lengua materna a la lengua meta (Schmidt y Richards, 1980; Thomas, 1983; Escandell Vidal, 1996). Llegados a este punto consideramos relevante distinguir entre interferencia pragmalingüística e interferencia sociopragmática. Thomas (1983: 101) se refiere a ambas como failure y define a las primeras como sigue:

Pragmalinguistic failure, which occurs when the pragmatic force mapped by $\mathrm{S}$ onto a given utterance is systematically different from the force most frequently assigned to it by native speakers of the target language, or when speech act strategies are inappropriately transferred from L1 to L2.

Por otra parte, Escandell Vidal entiende estas mismas como «el uso en una lengua de una fórmula propia de otra, con un significado del que carece en la primera» (1996: 99). Cuando un interlocutor neerlandófono A le dice a un hispanohablante B '¿Puedo (tener) una cerveza, por favor?' [Mag ik een bier, alstublieft? ${ }^{4}$ ], en realidad lo esperado en español sería ‘¿Me pones una cerveza, por favor?' y no una traducción literal de su lengua materna. En lo que a error sociopragmático se refiere, Thomas (1983: 99) se refiere a él como «sociopragmatic failure, a term I have appropriated from Leech (1983: 10-11), which I use to refer to the social conditions placed on language use. [...] sociopragmatic failure stems from cross-culturally different perceptions of what constitutes appropriate linguistic behaviour».

A su vez, Escandell Vidal (1996: 103) lo define como «el traslado a otra lengua de las percepciones sociales y las expectativas de comportamiento propias de otra cultura». Por ejemplo, ante un enunciado en forma de ofrecimiento como '¿te apetece un café?', diferentes culturas reaccionarían de manera distinta. Mientras que en la cultura anglosajona el interlocutor correspondiente reaccionaría contestando 'Sí, gracias' en la española sería de esperar la siguiente intervención reactiva 'no, gracias, no te molestes', cuando en realidad lo que espera este interlocutor es una reacción de insistencia antes de aceptar el ofrecimiento.

Si bien estos dos tipos de interferencia constituyen una de tipo pragmático, presentan diferencias. Mientras que los aspectos pragmalingüísticos tienden a presentar menos dificultades debido a que consisten en la transposición de una forma lingüística de la lengua materna a la lengua meta, los sociopragmáticos presentan un mayor obstáculo por encontrarse vinculados a un significado implícito convenido por unas expectativas socioculturales concretas y, consecuentemente, menos perceptibles para el alumno. En otras palabras,

Whereas pragmalinguistics (i.e., forms) appear to be learnable through implicit instruction in which attention is not intentionally directed to language form, it appears that explicit instruction (i.e., meta-pragmatic information is provided as a pedagogical focus) is necessary for learners to be able to connect form to sociopragmatic meaning. In other words, and at the risk of oversimplifying the issue, forms are easy to pick up, but sociopragmatic meanings are difficult to deduce without someone making the patterns visible to learner (Van Compernolle at al., 2016: $343)$.

La emisión de interferencias de esta naturaleza puede llevar el acto comunicativo a la deriva y fomentar la emisión de malentendidos, como apunta Trujillo Sáez (2005: 34):

\footnotetext{
${ }^{4}$ Traducción literal.
} 
Hay espacio para el éxito comunicativo aunque la comunicación también supone malentendidos puesto que se basa en la inferencia, la asignación de intenciones comunicativas y la interpretación. Sin estos procesos cognitivos, que pueden provocar ansiedad e incertidumbre, no hay comunicación.

En definitiva, ya no solo es cuestión de disminuir el número de malentendidos -ya que, al fin y al cabo, habría manera de solventarlos a través de otros recursos-, sino de evitar a nuestros estudiantes esa inseguridad que les puede provocar el desconocimiento de esas normas sociopragmáticas. Por todas estas cuestiones, una de nuestras responsabilidades docentes ha de ser la concienciación y gestión del CP por parte de nuestros estudiantes (Bardovi-Harlig, 1996). Del mismo modo, no podemos ignorar que

la enseñanza y aprendizaje de los contenidos culturales en el aula no estará orientada a que los alumnos lleguen a comportarse de igual manera que lo harían los nativos, reproduciendo sus comportamientos de forma mimética, sino a que sepan descodificarlos y comprenderlos (Guillén Díaz, 2004: 849)

Sin embargo, lo que es cierto es que la pragmática, cuando pensamos en aprender y enseñar una lengua, suele quedar aislada o relegada a un segundo plano por su complejidad, por su subjetividad y por la falta de conocimiento. De hecho, nuestra experiencia docente nos ha demostrado que, en una gran mayoría de los casos, se debe a la inseguridad o al desconocimiento por parte del docente sobre cómo integrarla o en qué medida. Por lo general, persiste el denominado 'efecto avestruz' que consiste en mirar hacia otro lado esperando, sin que ocurra, que el problema desaparezca, lo que fomenta la enseñanza de un español 'descafeinado', insulso y falto de realidad. En consecuencia, se produce un sabotaje inconsciente que se refleja en nuestra manera de dar clase, puesto que estamos determinando el alcance de lo que enseñamos, dejándonos cosas en el tintero, como ocurre con el CP.

¿A qué se debe esta falta de normalización e integración práctica del CP? Una de las principales causas es que la gran mayoría de los programas de las formaciones para ser profesor de $\mathrm{E} / \mathrm{LE}^{5}$ no ha dedicado un espacio concreto para enseñar a futuros profesores cómo integrarlo en el aula y se da preferencia a otros aspectos como la integración de las destrezas, cómo llevar la gramática y/o el léxico de manera motivadora, lo afectivo en el aula, el uso de las TIC, entre otros; en definitiva, aspectos incuestionablemente relevantes pero que, inconscientemente, otorgan un segundo lugar al que subyace a todos ellos: el $\mathrm{CP}^{6}$. A esto se suma la ausencia de herramientas didácticas reales que fomenten la aplicabilidad de este fenómeno en el aula, como acertadamente apuntan Gironzetti y Koike (2017: 90):

When faced with the complex task of teaching Spanish pragmatics, instructors have access to books, articles, and websites that purport to provide the information and resources necessary to accomplish this task. Unfortunately, the majority of these materials adopt a highly theoretical approach to pragmatics, failing to equip teachers with pedagogical tools needed to integrate pragmatics in the Spanish language classroom.

\footnotetext{
${ }^{5} \mathrm{Si}$ bien es cierto, esta situación está cambiando y la enseñanza del CP ya se empieza a integrar en cada vez más formaciones, sobre todo en másteres recientes y gracias a las infinitas posibilidades que nos ofrece la red por la gran acogida que tienen las formaciones en formato MOOC's. Para más información recomendamos la lectura del trabajo de De Pablos-Ortega (2016) y el de Níkleva (2018).

6 Concretamente, la situación en Flandes en Bélgica es un claro reflejo de esta situación en las formaciones $\mathrm{E} / \mathrm{LE}$, como presentamos en nuestra comunicación del pasado congreso XXXXXXX.
} 
A pesar de la existencia restringida de recursos y materiales, por lo general de acceso en línea, provenientes de Estados Unidos como los de la Universidad de Austin (https://coerll.utexas.edu/methods/), el Center for Advanced Research on Language Acquisition (CARLA, www.carla.umn.edu/index.Html) o los recursos de la Universidad de Indiana (http://www.indiana.edu/ discprag/) (De Pablos-Ortega, 2017: 172), los manuales y herramientas didácticas siguen siendo limitados y, sobre todo, simplistas a la hora de proporcionar una metodología clara y concreta para tratar el CP en el aula.

Más aún, otra de las piedras en el camino con la que nos topamos en la enseñanza del $\mathrm{CP}$ es dar respuesta a la simple y a la vez compleja pregunta de qué normas pragmáticas son más pertinentes enseñar:

\begin{abstract}
Should we attempt to teach heritage language learners monolingual Spanish pragmatics? And if so, which monolingual pragmatic norms are preferred? There is no clear answer to this question [...] They include expanding learners' communicative opportunities and reducing the risk of pragmatic inappropriateness when interacting with native Spanish speakers" (Gironzetti y Koike, 2017: 92)
\end{abstract}

$\mathrm{Su}$ respuesta es prácticamente imposible. El mundo hispanohablante es muy amplio y variado, lo que dificulta la delimitación de una tipología de convenciones pragmáticas. Pretender hacerlo nos conduciría a una deriva investigadora asegurada. Por ello, para el presente trabajo y desarrollo de la herramienta didáctica que presentamos a continuación en la siguiente sección, hemos tomado como pilares centrales dos ideas apuntadas por estos autores, a saber: (a) fomentar oportunidades comunicativas y (b) reducir el riesgo de enunciados pragmáticos inapropiados. A partir de ellas, hemos podido establecer los cimientos de un modelo que supone un puente hacia la aplicabilidad del CP y que destierre la imagen de oveja negra con tanta frecuencia asociada a la pragmática, en cuanto a lo que su enseñanza se refiere.

\title{
4. Una herramienta centrada en la aplicabilidad: el RICAI
}

En esta sección presentaremos el modelo RICAI que se caracteriza por su directa aplicabilidad en el aula y que pretende convertirse en un recurso práctico tanto para profesores como para aprendices. En lo que sigue, mostraremos los principales objetivos que persigue, la visión metodológica en la que se apoya y su planteamiento en el marco de explotaciones didácticas concretas.

\subsection{Objetivos y visión metodológica}

Nuestra herramienta, con el fin de facilitar la elaboración de actividades de enseñanza y de aprendizaje para la gestión del $\mathrm{CP}$ en $\mathrm{E} / \mathrm{LE}$, persigue los siguientes objetivos centrales:

1. Aumentar la conciencia pragmática de los estudiantes en el aula (BardoviHarlig, 1996).

2. Ayudar a los estudiantes a descodificar y comprender aspectos de naturaleza sociopragmática del español (Guillén Díaz, 2004: 849).

3. Disminuir el número de interferencias pragmáticas $\mathrm{y}$, de este modo, evitar situaciones comunicativas confusas y malentendidos (Thomas, 1983; Escandell Vidal, 1996). 
4. Motivar a los estudiantes para que descubran y aprendan a desarrollar una identidad intercultural.

5. Integrar y normalizar el $\mathrm{CP}$ desde un punto de vista didáctico en la práctica docente.

6. Gestionar el CP de una manera realista (procesual) y contextualizada con usos reales de la lengua dotados de autenticidad.

7. Desmitificar que la enseñanza del CP esté limitada exclusivamente a los niveles superiores y defender la enseñanza de aspectos pragmáticointerculturales desde el primer día en el aula.

Este recurso está concebido para facilitar y acomodar la enseñanza y adquisición de cualquier componente de naturaleza pragmática, con el fin de promover una adecuada gestión de este, a través de un proceso estructurado en cinco fases concretas, cuyas iniciales dan origen a su nombre (véase figura 1).

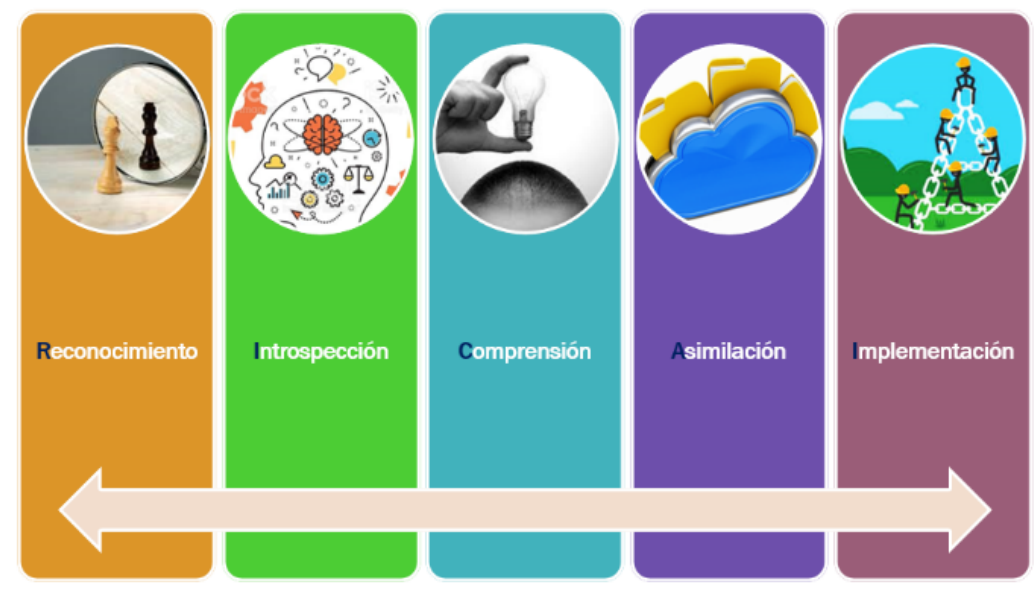

Figura 1. Herramienta RICAI (González Melón, 2017)

La principal razón de haber distinguido varias etapas radica en la dificultad que entraña la integración del CP dada su naturaleza, como hemos apuntado en las secciones anteriores. Consideramos prioritario hacerlo de manera procesual, puesto que aprender un elemento ajeno a las normas pragmático-sociales de nuestros aprendientes requiere un proceso que integre una serie de acciones o mecanismos que les permita desgranar y recopilar la información necesaria acerca del mismo.

Esta herramienta está diseñada para trabajar en un marco pedagógico como el que ofrecen los enfoques flipped classroom ${ }^{7} \mathrm{o}$ aquel basado en proyectos. Así, pretende centralizar el proceso de aprendizaje del CP en los propios alumnos, de modo que sean ellos mismos los que vayan construyendo su propia competencia intercultural en cada sesión. La razón de optar por contextos de enseñanza de este tipo y no por una versión clásica del aula tiene fundamentalmente que ver con la idea de 'optimizar' el aprendizaje. En primer lugar, las actividades llevadas a cabo y diseñadas por los docentes centran todos sus esfuerzos para que los aprendientes sean capaces de indagar, inicialmente, acerca del CP de manera autónoma - a través de una programación basada

\footnotetext{
${ }^{7}$ Enseñanza inversa, aula invertida en español.
} 
en un blended learning- pero continuamente supervisada. De esta manera, toman las riendas de su propio proceso de aprendizaje y se impulsa una implicación directa por parte del aprendiente. En segundo lugar, se aboga por la producción lingüística del estudiante durante las horas de contacto, de modo que aumentan las posibilidades de interacción entre ellos por medio de actividades centradas en estrategias de negociación, reflexión, retroalimentación, trabajos colaborativos, etc. Finalmente, este tipo de enseñanza fomenta un espacio sin interrupciones por parte del profesor con intervenciones centradas en meramente transmitir input instruccional (Kasper, 1997).

Así, a partir del enfoque del aula invertida, podemos trabajar diferentes aspectos de esa nueva norma pragmática en cada etapa por medio de la variación de formas de trabajo - como la autonomía en las fases iniciales-. De este modo, van incorporando conocimientos adquiridos que más tarde desarrollarán durante las horas de contacto a través de prácticas de colaboración o negociación. Cada una de estas fases comprenderá, por tanto, actividades que se realizarán o bien autónomamente en casa y en modo de aprendizaje combinado a modo de tareas $\operatorname{previas}^{8}$, o bien ya en el aula en forma de tareas posteriores ${ }^{9}$.

\subsection{Un proceso de cinco fases}

Para presentar la herramienta RICAI nos basamos en la siguiente secuenciación didáctica (véase figura 2) que nos sirve para ejemplificar y situar las cinco fases del proceso a través de una práctica docente concreta, a saber: el tabú lingüístico en $\mathrm{E} / \mathrm{LE}^{10}$.

TAREAS PREVIAS (CASA)

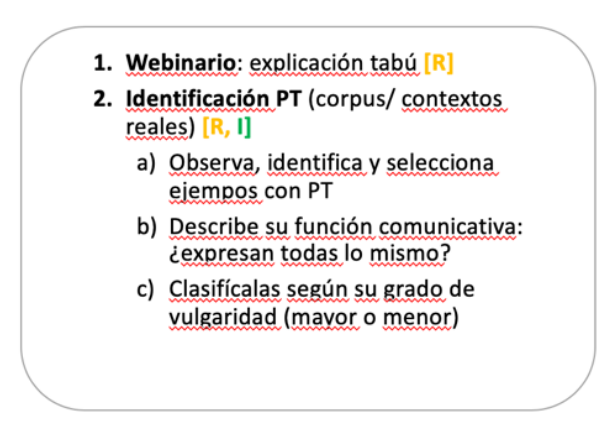

TAREAS POSTERIORES (AULA)

3. Interacción e intercambio (en grupos) $[C, A]$ ¿Existen palaras similares en tu LM? ¿Hay alguna que sea igual? ¿Son siempre tabú? Explica situaciones en las que:

a) no es aceptable usarlas

b) es posible usarlas

4. Negociación [C, A]:

a) Mirad estas situaciones $y$ votad la forma adecuada de reaccionar [p.ei. Kahoot, Socrative, Mentimeter]

5. Improvisación: reacciona usando una de estas expresiones (de pie, caminando) [I] y retroalimenta de manera conjunta

Figura 2. Secuenciación didáctica basada en el RICAI

\subsubsection{Fase de reconocimiento $(R)$}

Antes de pretender que nuestros alumnos aprendan una norma pragmática, sin más, ajena a la suya, han de poder reconocerla. Esta fase inicial de reconocimiento pretende

\footnotetext{
${ }^{8}$ Bajo las que se encuentran las etapas de reconocimiento e introspección.

${ }^{9}$ Que engloban las fases de comprensión, asimilación e implementación.

${ }^{10}$ Es necesario destacar que, basarnos en una explotación de un componente léxico para a un nivel medio o avanzado, no significa que esta herramienta sea exclusivamente representativa de estos niveles, pudiendo indudablemente adaptarse a actividades del nivel inicial, como adicionalmente demostraremos con diferentes actividades para cada fase.
} 
que el estudiante establezca una primera toma de contacto y digiera en qué consiste esa regla pragmática, que observe su forma lingüística, su composición y su estructura. En esta etapa el aprendiente opera siempre de manera autónoma e independiente en base a unas tareas previas diseñadas por el docente en una plataforma online. En ella, se produce un primer acercamiento individual a un determinado $\mathrm{CP}$ que promueve la contemplación de la existencia de nuevas normas pragmáticas.

Para ello, el docente parte de una planificación de actividades en formato TIC webinarios, fragmentos audiovisuales, etc.- que presentan al aprendiente ese CP de manera teórica, explicativa o ejemplificadora. Si retomamos la figura 2 -ahora simplificada como 2.a- podemos observar cómo se introduce el tema del tabú léxico a través de una primera actividad inicial marcada por la $[\mathrm{R}]$ y que hace referencia a esta fase. Por medio de un webinario explicativo, el profesor presenta la problemática asociada a este tipo de léxico, a qué palabras se refiere y la carga ofensiva que se les atribuye, a pesar de la alta frecuencia de uso por parte de algunos hispanohablantes, como es el caso de España.

\section{TAREAS PREVIAS (CASA)}

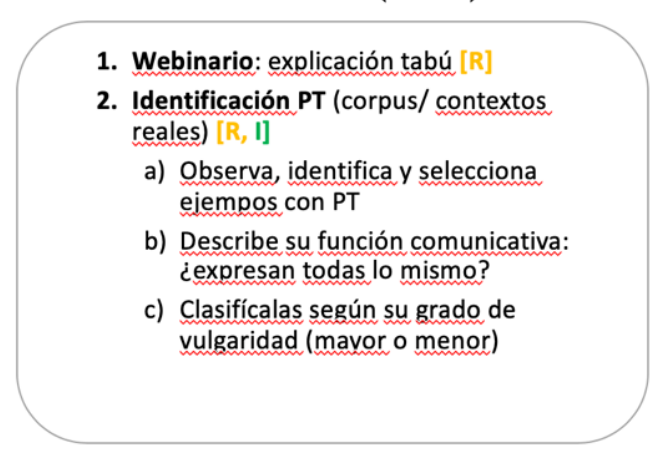

Figura 2.a. Fases de reconocimiento [R] y de implementación [I]

En una segunda actividad complementaria, el profesor pretende que los estudiantes identifiquen palabras tabú (PT en figura 2.a) a partir de, por ejemplo, corpus en línea ${ }^{11,}$ donde podrán observar su forma así como ser capaces de identificarlas en los contextos en los que aparecen ${ }^{12}$, por ejemplo: entre comas, al principio de una intervención, acompañadas de rasgos prosódicos o no (exclamación), junto a un vocativo, etc.

Igualmente, podemos introducir CP desde el primer día de clase en niveles iniciales. Tomemos, por ejemplo, el tema de las presentaciones en un nivel A.1. No solamente deberemos tener en cuenta las diferentes fórmulas de saludos o el grado de formalidad impuestos por el contexto dado, sino también la comunicación no verbal. En este caso la gestión de la distancia física es un elemento de naturaleza pragmática que cobra un lugar importante en el desarrollo de una buena práctica intercultural en cuanto a los saludos se refiere. Como tareas previas con el fin de introducir este CP, el profesor

11 Los corpus sobre conversaciones coloquiales como el del grupo Val.Es.Co (http://www.valesco.es/\#/pages/cod_jtvjm74bvgkjtlhlx59/cod_dhd0gulhdujtlhmes5) son aptos para llevar a cabo este tipo de actividad, aunque igualmente podrían utilizarse otros como el CREA (https://corpus.rae.es/creanet.html), el Preseea (https://preseea.linguas.net/Inicio.aspx) o el Cola sobre el habla oral adolescente (http://www.colma.org).

12 En esta actividad se produce un solapamiento con la segunda fase del método que presentamos, como explicaremos en el siguiente punto dedicado a la etapa de introspección. 
puede proponer las siguientes actividades para trabajarlas en esta fase de reconocimiento:

1. Visionado inicial (a partir del segundo 52 hasta el minuto 1:14): https://www.youtube.com/watch?v=NLTDMB5TAlg

2. Cuestionario en Google:

a. ¿Se conocen estas personas? ¿Sí? ¿No? ¿Por qué?

b. ¿Cómo se saludan? ¿Los dos de la misma manera, o hay diferencias?

Lista las maneras de saludarse que veas (explica qué hacen).

c. ¿Te sorprende el contacto físico en estas maneras de saludar?

d. ¿Conoces otras maneras de saludar en el mundo hispanohablante?

Por medio de un video humorístico se presenta un contexto concreto en el que la gestión del espacio físico entre ellos es un indicador directo de la relación que mantienen esas personas. Se lleva a cabo, así, un ejercicio previo de observación en el que el aprendiente, en combinación con un cuestionario posterior, identifica y reconoce no solo fórmulas de saludo sino también una manera de gestionar el contacto físico específico propio de esas situaciones comunicativas.

\subsubsection{Fase de introspección (I)}

Una vez que nuestros aprendientes ya han tomado contacto con esta norma pragmática y han dedicado un tiempo a reconocerla y a identificarla, pueden pasar a reflexionar sobre la realización de sus funciones pragmáticas (actos de habla) y sus efectos tanto en su lengua materna como en español. El objetivo central aquí es que el estudiante sea capaz de examinar y analizar qué funciones se llevan a cabo y, sobre todo, qué intención tiene un interlocutor al utilizar esta norma sociopragmática concreta. A partir de fragmentos de uso real de la lengua dotados siempre de autenticidad, el docente propone una actividad que permita al estudiante realizar esta introspección autónomamente.

En el marco de la secuenciación didáctica del tabú léxico (véase figura 2.a de nuevo), esta fase se lleva a cabo por medio de un análisis que parte de una observación de contextos reales y que aparece identificada por una [I] de introspección. El aprendiente tiene acceso a una serie de corpus ${ }^{13}$ que le sirven de herramienta para seleccionar libremente un número de ejemplos que contengan formas léxicas tabú. A partir de esa selección y teniendo en cuenta el contexto, tiene que intentar descubrir la función pragmático-comunicativa que cumplen (enfado, sorpresa, halago, entre algunas de ellas) y reflexionar sobre las diferentes funciones pragmáticas que realizan. A continuación, clasifica sus ejemplos en base a un mayor o menor grado de ofensa, según la intención que crean comunicada en ese contexto dado. Tras esta reflexión planteada en forma de tres pequeñas actividades, el aprendiente es capaz de realizar por sí mismo una exploración, de recapacitar sobre lo que ocurre cuando utilizamos una palabra tabú y, en definitiva, de tener una visión más clara del uso de expresiones malsonantes en español.

\footnotetext{
${ }^{13}$ Cualquiera de los indicados anteriormente o para un nivel superior: Madsex, un corpus de entrevistas sociolingüísticas de Madrid (Pizarro Pedraza, 2018).
} 
Si trasladamos esta fase de introspección a los niveles iniciales, vemos que igualmente se puede realizar una reflexión sobre cualquier componente de la lengua con una extensión pragmática del mismo. Pongamos por ejemplo el imperativo en un nivel A2. Si pedimos a nuestros estudiantes antes de venir a clase que realicen una tarea para que reflexionen acerca del uso del imperativo a partir de un número de fragmentos escritos en forma de entradas en Twitter, por ejemplo, fomentamos en el estudiante un acto de reflexión real, contextualizando el tema a estudiar y haciéndolo significativo para ellos. De esta manera, les pedimos que identifiquen enunciados en imperativo y que los clasifiquen según el acto de habla que realizan para poder recapacitar sobre las diversas funciones pragmáticas que cumplen. Esta reflexión permite al estudiante entender las limitaciones que acarrea la clásica asociación 'imperativo $=$ comando, orden' y que existen muchos casos en los que se utiliza para expresar tanto invitaciones, como ofrecimientos, sugerencias o incluso peticiones. Así, dotamos a los aprendientes de una mochila de consideraciones que les permitirá gestionar mejor el $\mathrm{CP}$ vinculado al uso de un imperativo en las fases siguientes.

\subsubsection{Fase de comprensión (C) y asimilación (A)}

La fase de comprensión, junto a la de asimilación ${ }^{14}$, anuncia el ecuador en este proceso de aprendizaje del CP e indica una transición en las estrategias que nuestros estudiantes van a desarrollar. A partir de este momento, las actividades se llevan al aula a través de la variación y la variedad de nuevas formas de trabajo motivadoras que promueven el aprendizaje colaborativo. En este punto, los aprendientes ponen en funcionamiento durante las horas de contacto lo que han identificado y sobre lo que han reflexionado hasta ahora de manera autónoma acerca de una norma sociopragmática. Es decir, se realiza una maniobra de comprensión que prepara los cimientos para un entendimiento adecuado de esa norma y una posterior asimilación de esta. En un primer momento es necesario comprender cómo es la norma pragmática aprendida desde la realidad de cada estudiante, para luego fomentar una asimilación de esta en la lengua meta. Por medio de actividades de aprendizaje centradas en la interacción entre los aprendientes, el docente elabora una serie de tareas que estimulan la práctica basada en estrategias de intercambio y de negociación con el fin de promover un intercambio para comprender, aceptar y empezar a integrar un CP específico en la lengua extranjera.

En nuestra secuenciación didáctica sobre el tabú léxico (véase figura 2.b), estas dos fases se traducen a modo de actividades -indicadas por una [C] y una [A]- en las que cada estudiante intercambia información con sus compañeros sobre la realidad de esa norma pragmática en su propia cultura y, así, promover una perspectiva intercultural en el aula (figura 2.b, sección 3). Partiendo de sus propias normas sociopragmáticas, los estudiantes intercambian modelos o pautas de expectativas de esta naturaleza que les permitirán asentar y comprender mejor la nueva norma que están aprendiendo. A continuación, se plantean actividades de negociación suplementarias para asegurar la comprensión y una adecuada asimilación del CP estudiado (figura 2.b, sección 4), a través de una votación o concurso con una TIC.

\footnotetext{
${ }^{14}$ Explicamos la tercera y la cuarta fase de manera conjunta por su estrecha relación ya que representan dos pasos complementarios en un proceso de entendimiento, es decir, tenemos que comprender algo antes de poder asimilarlo.
} 
TAREAS POSTERIORES (AULA)

3. Interacción e intercambio (en grupos) $[\mathrm{C}, \mathrm{A}]$ ¿Existen palaras similares en tu LM? ¿Hay alguna que sea igual? ¿Son siempre tabú? Explica situaciones en las que:

a) no es aceptable usarlas

b) es posible usarlas

4. Negociación [C, A]:

a) Mirad estas situaciones y votad la forma adecuada de reaccionar [p.ei. Kahoot, Socrative, Mentimeter]

5. Improvisación: reacciona usando una de estas expresiones (de pie, caminando) [I] y retroalimenta de manera conjunta

Figura 2.b. Fases de comprensión [C], asimilación [A] e implementación [I]

Estas dos fases se pueden llevar del mismo modo a niveles iniciales como, por ejemplo, el acto de rechazar una invitación y, por extensión, el uso de la negación en español. Consideremos el diálogo de (5) contextualizado a modo de una invitación por parte de los padres de tu mejor amigo/tu novio, de tu jefe a su casa, de un compañero en tu trabajo nuevo, etc. En definitiva, un contexto que representa la primera vez o de las primeras veces que alguien, a quien no conoces mucho o con el que no tienes una excesiva confianza, te invita a su casa:

(1) A- ¿Te apetece un aperitivo?

B- No, gracias, no es necesario/ te molestes ${ }^{15} \ldots$

A- ¿No? ¿Seguro? ¿No quieres nada? ¿Una copita de vino? ¿Cerveza?

B- No, no, de verdad, es que acabo de tomarme una...

A- Que sí, venga va, una cortita, anímate

B-

Lo interesante de este tipo de diálogos es que permiten mostrar al aprendiente dos tipos de expectativas sociopragmáticas: por un lado, la manera de aceptar o rechazar una invitación en ese contexto dado y, por otro, lo que se espera de él como anfitrión cuando extienda una futura invitación a alguien con el que mantiene ese tipo de relación y familiaridad. Si nos centramos en la primera de ellas - puesto que estamos enseñando la negación y por extensión el acto de rechazar una invitación- podemos elaborar actividades de aprendizaje que fomenten la comprensión de maneras de reaccionar ante situaciones comunicativas similares a partir de su propia cultura. A partir de estrategias de intercambio y de negociación, los estudiantes asimilarán la existencia de diferentes maneras de reaccionar ante dicha situación y adquirirán nuevas habilidades para asimilar esa norma pragmática ajena (o no) a la suya propia e incorporarla a su sistema sociopragmático.

\subsubsection{Fase de implementación (I)}

La última fase de nuestra herramienta persigue la puesta en práctica de una norma sociopragmática concreta. Vendría a ser la etapa en la que, idóneamente, serían capaces

\footnotetext{
${ }^{15}$ Dependiendo del nivel A.1 o A.2.
} 
de implementar dicha norma y utilizarla correctamente en unas coordenadas contextuales dadas por el profesor de antemano.

En el marco de la unidad didáctica sobre el tabú léxico (véase de nuevo la figura 2.b), el docente presenta actividades de aprendizaje que promueven la implementación indicada como [I]- de una norma sociopragmática para comprobar que saben utilizarla. En este caso concreto, han de levantarse, caminar por el aula y reaccionar utilizando algunas de las expresiones lingüísticas trabajadas en base a diferentes situaciones comunicativas que les presentan algunos de sus compañeros. A continuación, se cambian los roles y se procede de la misma manera. Igualmente, en un nivel inicial la fase de implementación permite el mismo tipo de práctica en al aula. Para finalizar, esta etapa se combina con un cierre final de carácter retroalimentador donde los aprendientes evalúan conjuntamente la práctica llevada a cabo con el fin de intercambian impresiones, presentar dudas e introducir mejoras en caso necesario.

\section{Conclusiones}

Nuestro principal cometido en este trabajo ha sido arrojar luz a uno de los obstáculos todavía presentes el aula de E/LE: la enseñanza del componente pragmático $(\mathrm{CP})$. Si, como bien se recoge en el MCER, tenemos la responsabilidad de hacer que nuestros aprendientes sean competente interculturalmente, no podemos mirar hacia otro lado ni dejar de tratarlo en el aula.

A pesar de la literatura existente y de los esfuerzos por parte de los expertos a la hora de ofrecer pistas y reflexionar sobre posibles maneras de introducirlo en nuestras planificaciones, la falta de recursos que faciliten su aplicabilidad sigue estando a la orden del día. Por este motivo, uno de nuestros objetivos centrales ha sido presentar una herramienta que ofrezca un soporte didáctico a la comunidad docente: el RICAI. Hemos podido mostrar y explicar su funcionamiento con secuenciaciones didácticas concretas y aplicables, tanto a niveles iniciales como avanzados dentro del marco de la enseñanza inversa. En base a este modelo, pensado como un proceso de cinco fases para una adecuada introducción de las normas sociopragmáticas de E/LE, animamos a gestionar el componente pragmático integrándolo de manera consciente y realista. Creemos igualmente necesario destacar que, en ocasiones y dependiendo del nivel en el que se encuentren nuestros estudiantes, no es tan importante saber utilizar e implementar correctamente normas de esta naturaleza; en ocasiones, basta con saber reconocerlas para evitar confusiones y malentendidos comunicativos. El RICAI, gracias a la forma en la que está concebido, permite una gestión procesual del $\mathrm{CP}$ y promueve la concienciación de la importancia de las expectativas sociopragmáticas en la enseñanza y el aprendizaje de E/LE, cuyo fin último es el de hacer del aprendiente uno intercultural.

\section{Bibliografía}

Álvarez, Alfredo, DE la Hoz, Concha, BARRIENTos, Laura, IGLESIAS, Isabel, BRAFIA, Mónica, Martínez, Pablo, Coto, Vanessa, Prieto, María, Cuevas, Miguel y TurzA, Ana (eds.) 2006. «La competencia pragmática y la enseñanza del español como lengua extranjera», Actas del XVI Congreso Internacional de ASELE (Asociación para la Enseñanza del Español como Lengua Extranjera). Oviedo: Servicio de Publicaciones de 
la Universidad de Oviedo. Recuperado el 12/3/20, de: http://cvc.cervantes.es/ensenanza/biblioteca_ele/asele/asele_xvi.htm

Amenós Pons, José., Ahern, Aoife, y Escandell VidAl, María Victoria. 2019. Comunicación y cognición en ELE: la perspectiva pragmática. Madrid: Edinumen.

BARdOvi-HARlig, Kathleen, HARTFORD, Beverly S., Mahan-TAYlor, Rebecca, MorGAn, Mary J., Y REYNOLDS, Dudley W.. 1991. «Developing pragmatic awareness: Closing the conversation», ELT Journal, 45 (1), 4-15.

BARDOVI-HARLIG, Kathleen Y MAHAN-TAYLOR, Rebecca. 2003. Teaching Pragmatics. Washington, DC: United States Department of State.

Betancourt ROMERO, María Victoria. 2012. Adquisición de pragmática en segunda lengua: Un modelo didáctico para la enseñanza de la pragmática. TFM, Indiana University. Recuperado el 12/7/20, de: https://scholarworks.iupui.edu/bitstream/handle/1805/2968/MV\%20Romero\%20TESIS. pdf? sequence $=1 \&$ isAllowed $=y$

COLA corpus. DOI: http://www.colma.org

CONSEJO DE EUROPA. 2002. Marco común europeo de referencia para las lenguas: aprendizaje, enseñanza, evaluación. Madrid: Secretaría General Técnica del MEC, $\begin{array}{llll}\text { Anaya } & \text { Instituto Cervantes. }\end{array}$ http://cvc.cervantes.es/ensenanza/biblioteca_ele/marco

CREA corpus. DOI: https://corpus.rae.es/creanet.html

De Pablos-Ortega, Carlos. 2017. «Pragmática en la formación de profesores de español como segunda lengua», Journal of Spanish Language Teaching 3 (2), 171-188. DOI: https://doi.org/10.1080/23247797.2016.1251783

ESCANDELL VIDAL, María Victoria. 1996. Introducción a la pragmática. Barcelona: Ariel.

FÉLIX-BrasDefer, César, J. 2007. «Pragmatic Development in the Spanish as a FL Classroom: A Cross-sectional Study of Learner Requests», Intercultural Pragmatics 4 (2), 253-286.

FÉLIX-BRASDEFER, César, J. 2008. «Teaching Pragmatics in the Classroom: Instruction of Mitigation in Spanish as a Foreign Language», Hispania 91(2), 479.

FÉLIX-BRASDEFER, César, J. 2018. Pragmática del español: contexto, uso y variación. Londres: Routledge.

FÉliX-Brasdefer, César, J. Y. Cohen, Andrew D. 2012. «Teaching Pragmatics in the Foreign Language Classroom: Grammar as a Communicative Resource», Hispania 95 (4), 650-669.

FÉliX-Brasdefer, César, J. Y KoIKe, Dale. 2014. «Perspectives on Spanish Second Language Acquisition from Pragmatics and Discourse». En M. Lacorte (ed.), The Routledge Handbook of Hispanic Applied Linguistics. Londres: Routledge, 25-48.

FERNÁNDEZ SILVA, Claudia. 2002. «La programación de cursos y el desarrollo de la competencia pragmática», Comunicación/ponencia/curso presentado/leído en el XI Encuentro práctico de profesores de ELE. Barcelona, 1-10. DOI: https://www.encuentro-practico.com/pdf/cfernandez2.pdf 
GIRONZETTI, Elisa. Y KOIKE, Dale. 2017. «Bridging the gap in Spanish instructional pragmatics: from theory to practice/Acortando distancias en la enseñanza de la pragmática del español: de la teoría a la práctica», Journal of Spanish Language Teaching 3 (2), 89-98. DOI: https://doi.org/10.1080/23247797.2016.1251781

GUILLÉN DíAZ, Carmen. 2004. «Los contenidos culturales». En J. Sánchez Lobato y I. Santos Gargallo (dirs.), Vademécum para la formación de profesores. Enseñar español como segunda lengua (L2) / lengua extranjera (LE), 835-851. Madrid: SGEL.

GonZÁlez Melón, Eva. 2017. «¿Nos dejamos algo en el tintero? Acerca del tratamiento de la pragmática en la formación de profesores de ELE», Comunicación/ponencia/curso presentado/leído en el XXVIII Congreso Internacional ASELE, Tarragona, 5-9 de septiembre de 2017.

HYMES, Dell. 1971. «Sociolinguistics and the ethnography of speaking». En E. Ardener (ed), Social anthropology and language, 47, 93. Londres: Routledge.

InStituto Cervantes. 2006. Plan curricular del Instituto Cervantes. Niveles de referencia para el español. Madrid: Instituto Cervantes y Biblioteca Nueva

INSTITUTO CERVANTES. 2012. «Las competencias clave del profesorado de lenguas segundas y extranjeras». DOI: https://cvc.cervantes.es/ensenanza/biblioteca_ele/competencias/competencias_profesora do.pdf

KASPER, Gabriele. 1997. «Can pragmatic competence be taught?». DOI: http://www.nflrc.hawaii.edu/NetWorks/NW06/

LEYOU, Fan. 2015. Unidad didáctica de nivel A1 para suplir las carencias en la competencia pragmática en ELE del manual Español Moderno. Tesis fin de máster inédita. Universidad de Valladolid]. https://repository.javeriana.edu.co/handle/10554/17103

NíkLEVA, Dimitrinka Georgieva. 2018. Necesidades y tendencias en la formación del profesorado de español como lengua extranjera. Frankfurt am Main: Peter Lang.

PinTO, Derrin. Y De PABlos-ORTEGA, Carlos. 2014. Seamos pragmáticos: introducción a la pragmática española. New Haven y Londres:Yale University Press.

PiZARro PedraZA, Andrea. 2019. «MadSex: collecting a spoken corpus of indirectly elicited sexual concepts», Language Resources and Evaluation 53, 191-207. DOI: https://doi.org/10.1007/s10579-018-9435-x

PONS BORDERÍA, Salvador. 2005. La enseñanza de la pragmática en la clase de E/LE. Madrid: Arco Libros.

PRESEEA corpus. DOI:https://preseea.linguas.net/

SCHMIDT, Richard. Y RICHARDS, Jack C. 1980. «Speech acts and second language learning», Applied Linguistics 1 (2), 129-57.

SIEBOLD, Kathrin. 2008. Actos de habla y cortesía verbal en español y en alemán: estudio pragmalingüistico e intercultural. Frankfurt am Main: Peter Lang.

SHIVELY, Rachel. 2011. «L2 pragmatic development in study abroad: A longitudinal study of Spanish service encounters», Journal of Pragmatics 43, 1818-1835. 
TAGUCHI, Naoko. 2009. Pragmatic Competence. Berlin: Mouton de Gruyter.

TAGUCHI, Naoko. 2011. «Teaching pragmatics: trends and issues», Annual Review of Applied Linguistics 31, 289-310.

THOMAS, Jenny. 1983. «Cross-cultural pragmatic failure», Applied Linguistics 4 (2), 91112.

TrujiLlo SÁEZ, Fernando. 2005. «En torno a la interculturalidad: reflexiones sobre cultura y comunicación para la didáctica de la lengua», Porta Linguarum 4, 23-29.

Toledo VegA, Gloria. 2012. Adquisición pragmática en español como lengua extranjera. Tesis doctoral inédita. Universidad de Valladolid.

VAL.Es.Co. 2021. Corpus Val.Es.Co 3.0. En http://www.valesco.es/\#/pages/cod_jtvjm74bvgkjtlhlx59/cod_dhd0gulhdujtlhmes5

VAN Compernolle, Rémi. A. 2014a. Sociocultural theory and L2 instructional pragmatics. Bristol: Multilingual Matters.

VAN COMPERnOlle, Rémi A. y Henery, Ashlie. 2014b. «Instructed concept appropriation and L2 pragmatic development in the classroom», Language Learning 64, 549-578.

Van Compernolle Rémi A., GómeZ-Laich Maria Pia, y Weber Ashley. (2016). «Teaching L2 Spanish Sociopragmatics Through Concepts: A Classroom-Based Study», The Modern Language Journal 100 (1), 341-361. DOI: https://onlinelibrary.wiley.com/doi/full/10.1111/modl.12318

Vera Luján, Agustín. y Blanco Rodríguez, Mercedes. 2014. Cuestiones de pragmática en la enseñanza de español como segunda lengua. Madrid: Arco/Libros. 